\section{Validation of Leaf Design Characteristics of an Add-on Automated Multi Leaf Collimator for Telecobalt Therapy Machine}

\author{
Roopa Rani Akula1,2,3® Komanduri Ayyangar4, Alla Ramalinga \\ Reddy ${ }^{4}$, Ayyalasomayajula Anil Kumar ${ }^{5}$, Pal Reddy Yadagiri \\ Reddy ${ }^{6}$
}

\begin{abstract}
Background: In developing countries like India, cobalt-60 machines still find their applicability, considering the cost and maintenance issues. With a view to deliver conformal treatment plans using teletherapy machines, an automated Multi-Leaf Collimator (MLC) was developed for the existing machines as a retrofit attachment to the collimator assembly without any modifications to the unit.

Objective: This study aims to investigate the radiation characteristics of leaf designs incorporated in two add-on prototype MLC systems with respect to the shape of leaf projected at the isocenter plane and the isodose distribution around the target. Besides, the dosimetric characteristics of prototype MLC with divergent leaf design are validated through simulation and experimental measurements.

Material and Methods: In this experimental study, two add-on prototype MLC systems were designed and fabricated. The characteristic measurements of leaf designs incorporated in both the prototypes were carried out using Gafchromic films (GAF) and compared with Monte Carlo (MC) simulations. For divergent leaf design, beam profiles were obtained using Monte Carlo simulations which are complemented with the results obtained from measurements of radiochromic films and ionization chamber (IC) profiler. Dosimetric characteristics like radiation field width and beam penumbra were evaluated.
\end{abstract}

Results: The Monte Carlo simulated data are in agreement with experimental data from IC profiler as well as from Radiochromic films. The results of this study are well within acceptable tolerance limits.

Conclusion: The prototype MLC system designed for existing telecobalt machines supports its clinical applicability for conformal therapy to better manage treatment in rural areas, which can provide superior cost effective treatments.

Citation: Akula RR, Ayyangar K, Reddy AR, Kumar AA, Yadagiri Reddy PR. Validation of Leaf Design Characteristics of an Add-on Automated Multi Leaf Collimator for Telecobalt Therapy Machine. J Biomed Phys Eng. 2021;11(6):723-734 doi: 10.31661/jbpe-v0i0.2002-1073.

Keywords

Developing Countries; Monte Carlo Method; Cobalt-60; MLCs

\section{Introduction}

$\mathrm{I}$ $\mathrm{t}$ is well-known that, radiotherapy is one of the important modality in the treatment of patients suffering from different types of cancers- malignant or benign. It has emerged as one of the cost effective ways in cancer management considering patient stage and health status. The cancer treatments are performed by a wide range of radiation generating machines such as cobalt-60 machines and high energy linacs

${ }^{1} \mathrm{MSc}$, Department of

Physics, Osmania Uni-

versity, Hyderabad, India

${ }^{2} \mathrm{MSc}$, Department of

Radiation Oncology, MNJ

Institute of Oncology \&

Regional Cancer Center,

Hyderabad, India

${ }^{3} \mathrm{MSc}$, Department of

Radiation Oncology,

International Cancer

Centre, MGMMT, Bhima-

varam, India

${ }^{4} \mathrm{PhD}$, Department of

Radiation Oncology,

International Cancer

Centre, MGMMT, Bhima-

varam, India

${ }^{5} \mathrm{PhD}$, Department of

Radiation Oncology, Ma-

hatma Gandhi Cancer

Hospital \& Research

Institute, Visakhapat-

nam, India

${ }^{6} \mathrm{PhD}$, Department of

Physics, Osmania Uni-

versity, Hyderabad, India

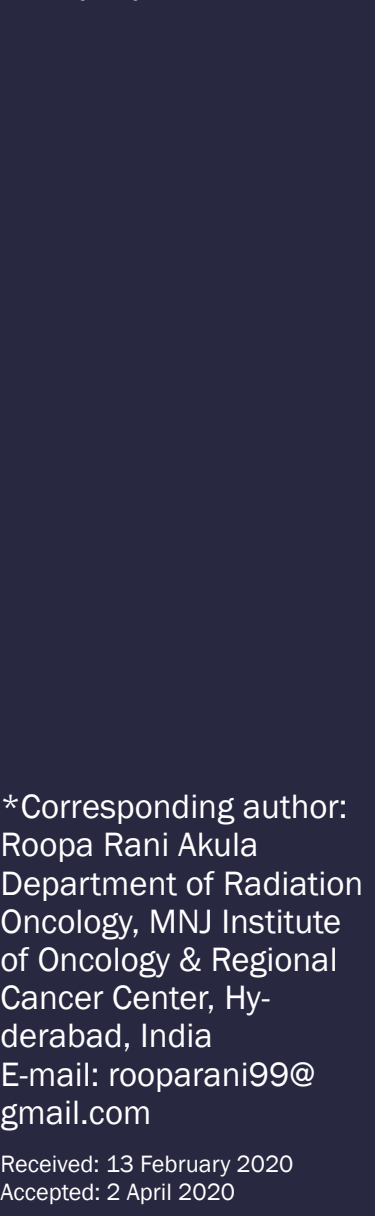


combined with advanced technologies. Different protocols are developed depending on the stage of cancer and presented for treatment to attain the goal of radiotherapy by minimizing radiation dose to normal tissues and to deliver maximum uniform dose to tumor by applying different strategic techniques.

According to $\mathrm{WHO}$, deaths due to cancers accounts to nearly $70 \%$ of the incidence rate in low- and middle-income countries. In developing countries like India, this increase in death rate is partly due to unavailability of appropriate centers for treatment and cost-effectiveness of required technology. Munshi et al. addressed the current situation of radiotherapy in India bridging the gap between past and present facilities with an overview for future developments [1]. In view of above, there is always a continuing need for affordable treatment machines considering the treatment facilities available for growing demands of cancer population. In addition to linear accelerators, cobalt-60 teletherapy machines still find their existence in terms of new advancements either through modifications to the existing ones or by development of indigenous machines incorporating new technological features [2].

Ravichandran emphasized that cobalt-60 machines still are better options for majority of treatable cancers in scenario of facilities available in radiation oncology departments in developing countries such as India [3]. Cobalt-60 machines with added beam improvements can be utilized for cost-effective treatment management. Van Dyke presented out the possibilities that how cobalt- 60 units can be improvised to provide dose distributions, comparable to those provided with higher energy radiation despite of its limitations compared to linear accelerators [4]. The multileaf collimators are one of those, eliminating the need for shielding blocks. They are a standard feature on accelerator machines; however, the telecobalt units can be retrofitted with modern multileaf collimator (MLC) owing to its characteristics.
Due to providing low-cost cancer care in rural areas of developing countries, continuing efforts are being made in the improvisation of cobalt- 60 beams for conformal therapy. A fully automated add-on MLC system integrated with $3 \mathrm{D}$ treatment planning system was developed for Theratron 780E phoenix telecobalt machine [5]. For this add-on MLC, there is no need to make any modifications to the machine. However, the add-on MLC limits us to use auxiliary tray holder and fits in the same space allocated for the block-tray slot that comes with the machine. Two prototypes, including prototype- 1 and prototype- 2 MLCs were designed and manufactured with different leaf designs and tested for their radiation characteristics.

In a study, Galvin et al. have reported that a dosimetric issue with MLC is the stepped dose pattern, occurring at the edges of the fields defined by MLC, where the leaves must be moved in order to conform to the irregular target which differs from the fields created by divergent block. Also, variations in daily patient setup have the advantage of blurring divergent block field edges and MLC field edges to some extent [6]. In another study, Galvin et al. suggested a different technique for removing the stepped dose that appears in the fields defined by MLC. This technique involves the movement of treatment-table to smoothen the stepped dose pattern at the MLC field edges [7]. The prototype MLC leaves in our study resulted in a smooth isodose lines across the fields despite of the irregular stepped pattern or smooth outline contour across the target. This is due to the relatively large penumbra caused by the cobalt- 60 source.

\section{Material and Methods}

In this experimental study, some of the characteristics of the two in-house developed addon MLCs were investigated.

Technical Design Specifications

The design and radiation characteristic study 
of prototype-1 MLCs were presented in conferences [8-10] and reported [5, 11]. For both the prototypes 1 and 2, the material used was tungsten alloy with density of $18 \mathrm{~g} / \mathrm{cm}^{3}$ (W $95 \%+\mathrm{Ni} 3.5 \%+\mathrm{Cu} 1.5 \%)$. The choice of tungsten alloy material for leaves was also suggested by AAPM report no.72 [12].

Leaf Configuration and Cross Section

To compare the leaf cross section, the prototype-1 comprises of 14 non-divergent leaf pairs that each leaf of $150 \mathrm{~mm}$ long with rounded edge, $7 \mathrm{~mm}$ wide by $40 \mathrm{~mm}$ height with straight edge bound through $20 \mathrm{~mm}$ height by $2 \mathrm{~mm}$ depth tongue and groove construction and defines a field size of $14 \times 14 \mathrm{~cm}^{2}$. It is important to find an optimal design for leaves because of the extensive use of MLCs in 3D treatment planning. In view of this necessity, prototype-2 was designed consisting of divergent 16 leaf pairs with $155 \mathrm{~mm}$ length, $40 \mathrm{~mm}$ height with $6.5 \mathrm{~mm}$ width on top and $7 \mathrm{~mm}$ width at the bottom to follow the divergence of the cobalt radiation with a step design for leaf sides and leaf end rounded edge. It can define a maximum field size of $14 \times 16 \mathrm{~cm}^{2}$ at the isocenter. Both the leaf pairs can overtravel a distance of $50 \mathrm{~mm}$ across the central axis. They are designed to project a leaf width of $10 \mathrm{~mm}$ at isocenter $(80 \mathrm{~cm})$.

The leaves are machined so that they follow beam divergence from a point source situated $52 \mathrm{~cm}$ from the top of the leaves and $56 \mathrm{~cm}$ from the bottom of the leaves.

\section{Automation of the Mlc}

The automation of prototype-2 MLC system, shown in Figure 1, differs from prototype-1 MLC system in terms of numbers of linear actuators needed to drive the respective leaves. In prototype-1, the leaves are driven by independent 28 linear actuator (LA) of 12 Volts each whereas in prototype-2, each leaf is driven by 2 LAs of 6 Volts each with different gear ratios. Additionally, the leaves were supported by ball bearings for smooth movement in the unused $50 \mathrm{~mm}$ length of the leaves. The leaf positions were calibrated to be reproduc-

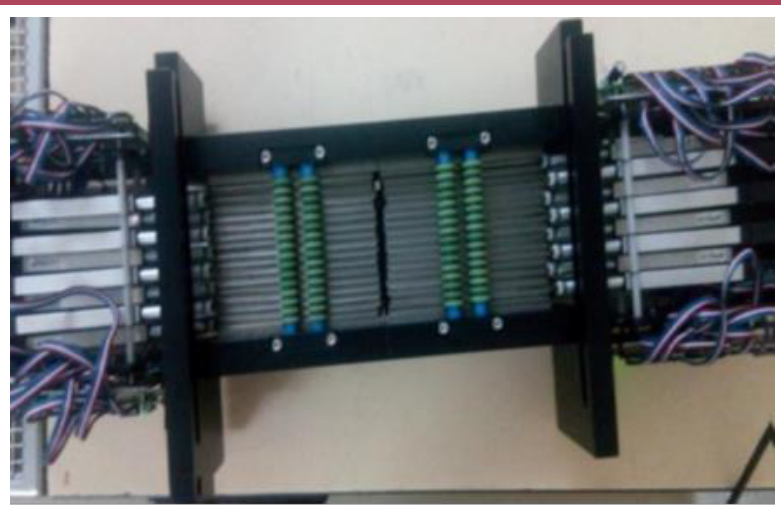

Figure 1: Prototype-2 Multi-Leaf Collimator (MLC) system

ible within 1-2 mm tolerance.

\section{Treatment Planning}

Both prototypes are integrated with $\mathrm{Ra}$ diation Oncology treatment planning system (ROPS) for conformal treatment plans. The detailed specifications of ROPS can be found from the website at https://sites.google.com/ site/tjcsrops. Publications on ROPS treatment planning system show feasibility in generating conformal plans for clinical radiation beams for different treatment machines [13, 14]. Three dimensional (3D) conformal planning requires an accurate delineation of the target volume and beam shaping to conform to the volume. The design of shaped fields is accomplished by multileaf collimator (MLCs) in each beam's eye view. The radiation field shaped by MLC has a stepwise outline and is not identical to the desired target field with smooth outline. When MLCs are used, the collimation occurs in discrete steps but the measurements indicate that the rounded edges of the MLC leaves resulted in a smooth outline. ROPS is facilitated to use both the options available, including smooth outline with margin around target by default or irregular field using MLC leaf shape for calculations. An irregular brain field planned with both features was exposed using Gafchromic film at isocenter and the re- 
sults are presented.

\section{Study of Leaf Designs}

To study the leaf designs, the projection of leaf shape at isocenter was evaluated [Figure 2]. In $6 \times 6 \mathrm{~cm}^{2}$ square field generated by MLCs, a single leaf was moved to the center of the field to study the effect of divergence.

The Monte Carlo (MC) simulations were carried out using the same machine specifications which were explained in detail in our previous simulations as well [11]. The MLC leaves were opened to $6 \times 6 \mathrm{~cm}^{2}$ square field with the collimator jaws wide open. One of the bank leaves of the field was positioned on the central axis. Each simulation had been run for 5 billion histories and the dose was calculated on a water phantom with DOSXYZnrc program. Planar data was extracted using separate programs and excel was used to evaluate the data.

For experimental verification, the study was carried out using Phoenix model Cobalt-60 machine at MNJ Institute of Oncology \& Regional Cancer Centre with Gafchromic (GAF) EBT3 films at the isocenter. The film was setup on virtual water slab at source to axis distance (SAD) of $80 \mathrm{~cm}$ with a buildup of 0.5 $\mathrm{cm}$ and irradiated for $1 \mathrm{~min}$. The images were scanned using EPSON 11000XL flatbed scanner and analyzed as well.

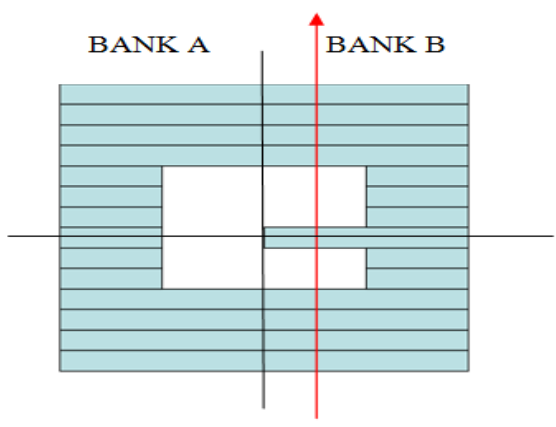

(A)
Study of Radiation Characteristics of Prototype-2 MlC

Extensive measurements were made using prototype-2 MLC to evaluate radiation properties.

\section{Transmission Measurements}

The transmission through the leaves and leaf end was determined with ion chamber measurements in air for the Theratron 780E Phoenix machine. A $0.13 \mathrm{~cm}^{3}$ ionization chamber was placed at $80 \mathrm{~cm}$ source to surface distance (SSD), measuring $24 \mathrm{~cm}$ from the bottom of the leaves on the central axis with $5 \mathrm{~mm}$ build cap. Measurements were made for $10 \times 10$ $\mathrm{cm}^{2}$ field defined by the MLC in the closed and open positions with the upper and lower jaws wide open. The ratio of measurements with MLC leaves closed to open gives the leaf end transmission.

For transmission through leaves, measurements were made by closing the leaves across the collimator field size at an off-axis point. The ratio of dose under closed field to that under open field, expressed in percentage, provides the transmission.

\section{Output Factors}

The ratio of output for a given field size to that for a reference field size gives the output factor. Measurements were taken for different collimators and MLC openings with a $0.13 \mathrm{cc}$ ion chamber with build cap of $0.5 \mathrm{~cm}$ setup in

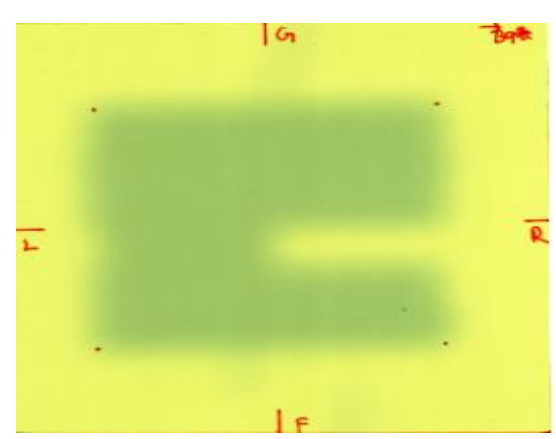

(B)

Figure 2: Single leaf projection at the center of field: (A) Block Diagram of Multi-Leaf Collimator (MLC) prototype (B) Gafchromic film with an exposed $6 \times 6 \mathrm{~cm}^{2}$ MLC field. 
air at $\mathrm{SAD}$ of $80 \mathrm{~cm}$. The output factors were normalized to the $10 \times 10 \mathrm{~cm}^{2}$ open field size.

\section{Dosimetric Parameters}

The Monte Carlo simulations were performed using BEAMnrc code with the full cobalt machine specifications, used in our previous simulations [11] with MLC prototype-2 at the block tray position. The source simulated used the bare Co-60 spectrum with cylindrical shape, emitting isotropic radiation. The phasespace files were generated at treatment distance of $80 \mathrm{~cm}$ from the source. These phasespace files from BEAMnrc simulation serve as the radiation source for dose deposition in a water phantom. The simulation process was run for $10^{10}$ histories for each field size. The parameters chosen were ECUT $=0.7 \mathrm{MeV}$ and PCUT $=0.01 \mathrm{MeV}$. The default EGSnrc transport parameters were used for accurate results. No variance reduction techniques were used. For each field size, dose deposition in $30 \times 30$ $\times 30 \mathrm{~cm}^{3}$ water phantom was simulated using DOSXYZnrc with $2.5 \mathrm{~mm}$ voxel size and compared against measured data.

Experimentally beam profiles were measured by IC Profiler ${ }^{\mathrm{TM}}$ (Sun Nuclear Corporation, Melbourne, FL) and Gafchromic EBT3 films. The commercial water scanners could not fit under the machine with MLC prototype attached to it due to clearance of $22 \mathrm{~cm}$ distance from MLC system to isocenter. Alternatively, IC profiler comprising of 251 multi-axis ion chamber arrays, each of $0.05 \mathrm{~cm}^{3}$ volume with a spacing of $5 \mathrm{~mm}$ from one detector to another, was used. Array calibration was done for the cobalt-60 beam with appropriate correction factors, including background correction of total $32 \times 32 \mathrm{~cm}^{2}$ active area of detector. Although, only the central $20 \times 20 \mathrm{~cm}^{2}$ area was used: this caters to our MLC generated field sizes. Measurements were performed in continuous mode with buildup of $1 \mathrm{~cm}$ adhering to its inherent buildup of $0.9 \mathrm{gcm}^{-2}$ at SAD of $80 \mathrm{~cm}$. The beam profiles were normalized and presented.

Similarly, EBT3 Gafchromic films were used with virtual water slabs to generate beam profiles. The films were calibrated for known doses at SAD $80 \mathrm{~cm}$ for a $5 \times 5 \mathrm{~cm}^{2}$ collimator field size, covering a dose range of $10 \mathrm{cGy}$ to $350 \mathrm{cGy}$. The prototype- 2 MLC generated fields, ranging from $2 \times 2$ to $14 \times 14 \mathrm{~cm}^{2}$ with fully open collimator, exposed in a SAD setup at $80 \mathrm{~cm}$ and at a depth of maximum dose. The films were cut $2 \mathrm{~cm}$ larger on both sides for each field and exposed. Precautions were taken for handling the films as per AAPM TG-55 report [15]. Using EPSON Expression 11000XL flatbed scanner, the films were scanned with 72 dpi resolution in 48-bit RGB mode. The profile data was taken directly from the dose matrix. Full width at half maximum (FWHM) and penumbra for each field size were evaluated.

\section{Results}

\section{Effect of Leaf Design}

The profile width was taken at $50 \%$ dose level. The profiles were taken at an off-axis distance of $1.5 \mathrm{~cm}$ from the center in the direction perpendicular to the leaf movement (Y-axis), as shown in Figures 3 and 4, for Monte Carlo simulations and Gafchromic film scans. Table 1 gives the comparison of leaf projection width obtained from Monte Carlo simulations and measurements obtained from Gafchromic films for prototype-1 and prototype-2 MLCs:

As seen in Table 1, penumbra effect is smaller with divergent leaf as expected.

\section{Treatment Planning Evaluation}

The Beam's-eye-view image will typically contain the images of the patient's anatomy and the beam modifiers such as jaws or MultiLeaf Collimator (MLC). The exposed irregular brain field is shown in the Figure 5.

\section{Radiation Characteristics of Pro- totype-2 MlC \\ Leakage through Mlcs}

The transmission measurements through the 


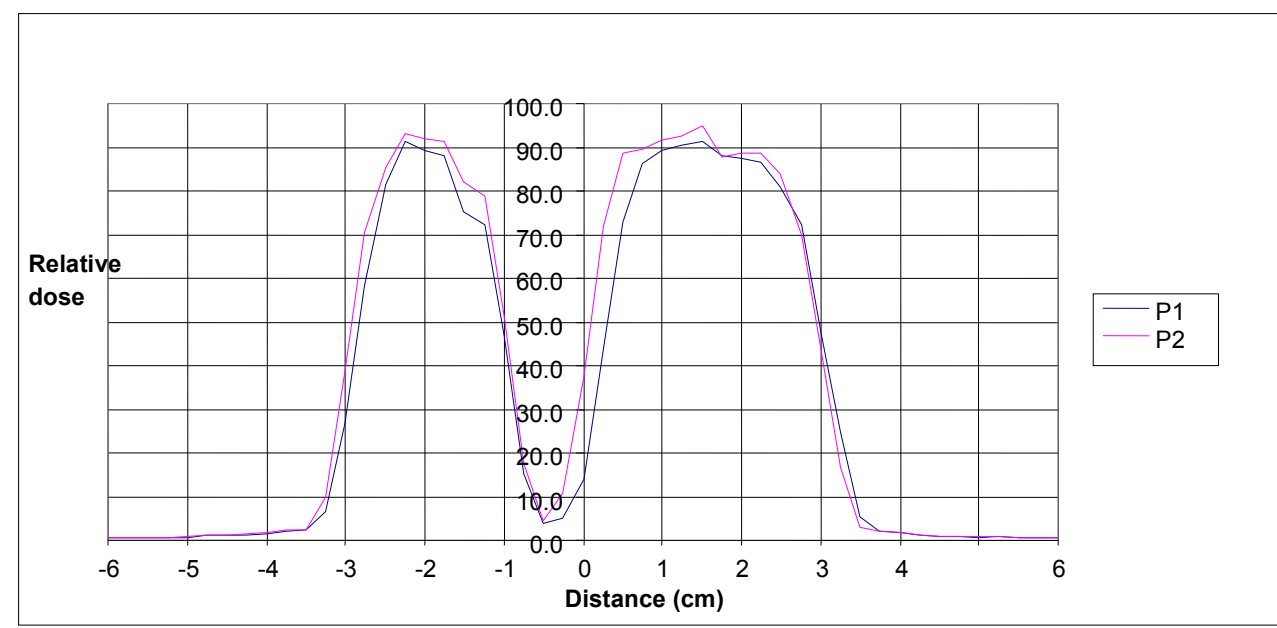

Figure 3: Profile width comparison at $1.5 \mathrm{~cm}$ distance from center through the middle of leaf for prototype-1 and prototype-2 Multi-Leaf Collimator (MLC) using Monte Carlo simulations.

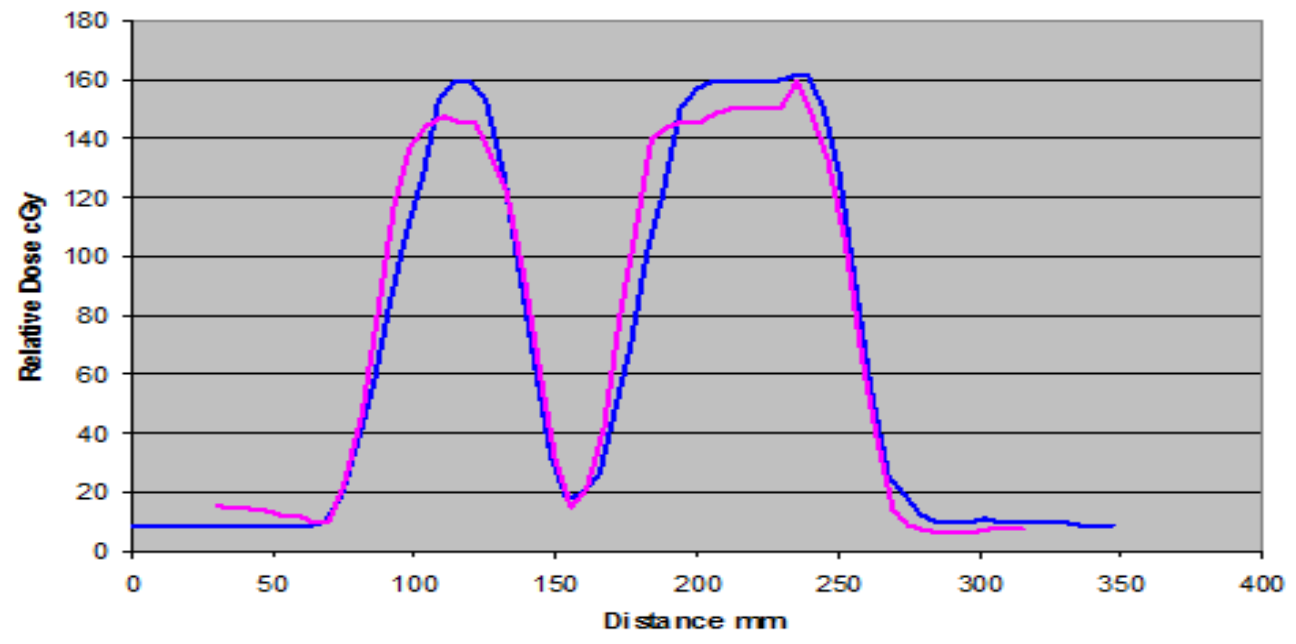

Figure 4: Profile width comparison at $1.5 \mathrm{~cm}$ distance from center through the middle of leaf for prototype-1 and prototype-2 Multi-Leaf Collimator (MLC) from Gafchromic film. Blue line represents prototype- 1 and magenta line represents prototype- 2 leaf widths.

Table 1: Comparison of leaf projection width of prototype Multi-Leaf Collimator (MLC) -1 and 2.

\begin{tabular}{ccc} 
FWHM & PROTO1 & PROTO2 \\
\hline MC Simulation & $1.25 \mathrm{~cm}$ & $1.01 \mathrm{~cm}$ \\
\hline GAF film & $1.35 \mathrm{~cm}$ & $1.05 \mathrm{~cm}$
\end{tabular}

FWHM: Full width at half maximum, MC: Monte Carlo, GAF: Gafchromic leaves indicated $1.6 \%$. The leaf end transmission is $3.7 \%$ which is in compliance with IEC recommendations compared to non-IMRT MLCs [16].

\section{Output Factors}

The treatment planning system uses a measured data file with appropriate MLC correction factors for accurate dose calculations. Figure 6 demonstrates the variation in output 


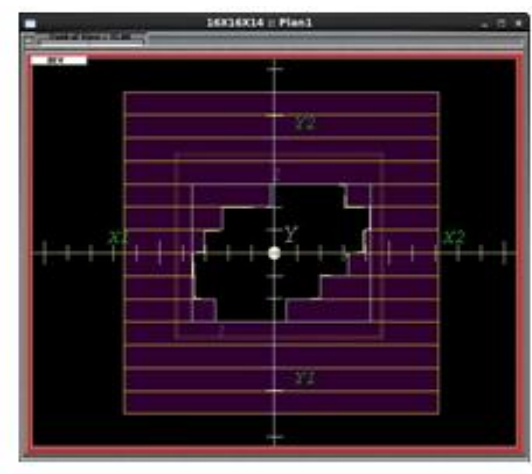

(A)

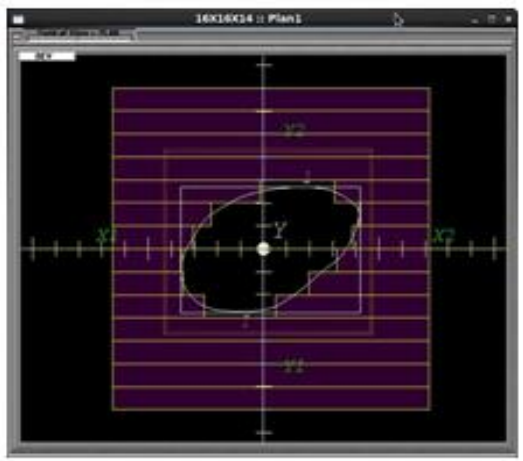

(C)

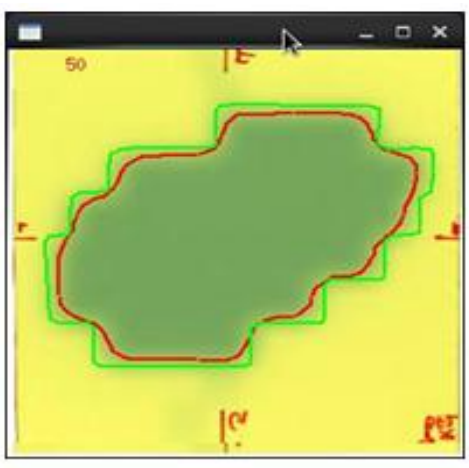

(B)

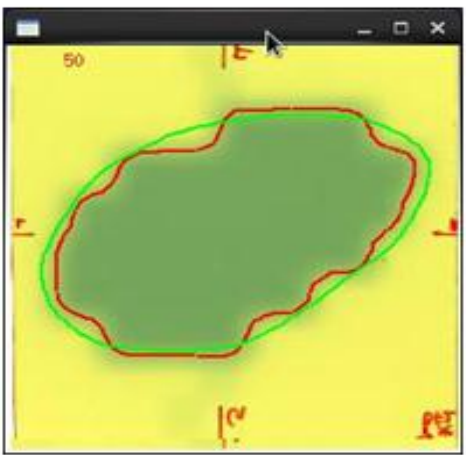

(D)

Figure 5: Stepwise outline (A) Beam's-eye-view (B) Dosimetric representation and Smooth outline (C) Beams eye view (D) Dosimetric representation in Radiation Oncology treatment planning system (ROPS). The red line depicts $50 \%$ isodose line from measured data and green line depicts Multi-Leaf Collimator (MLC) leaf position outline around target.

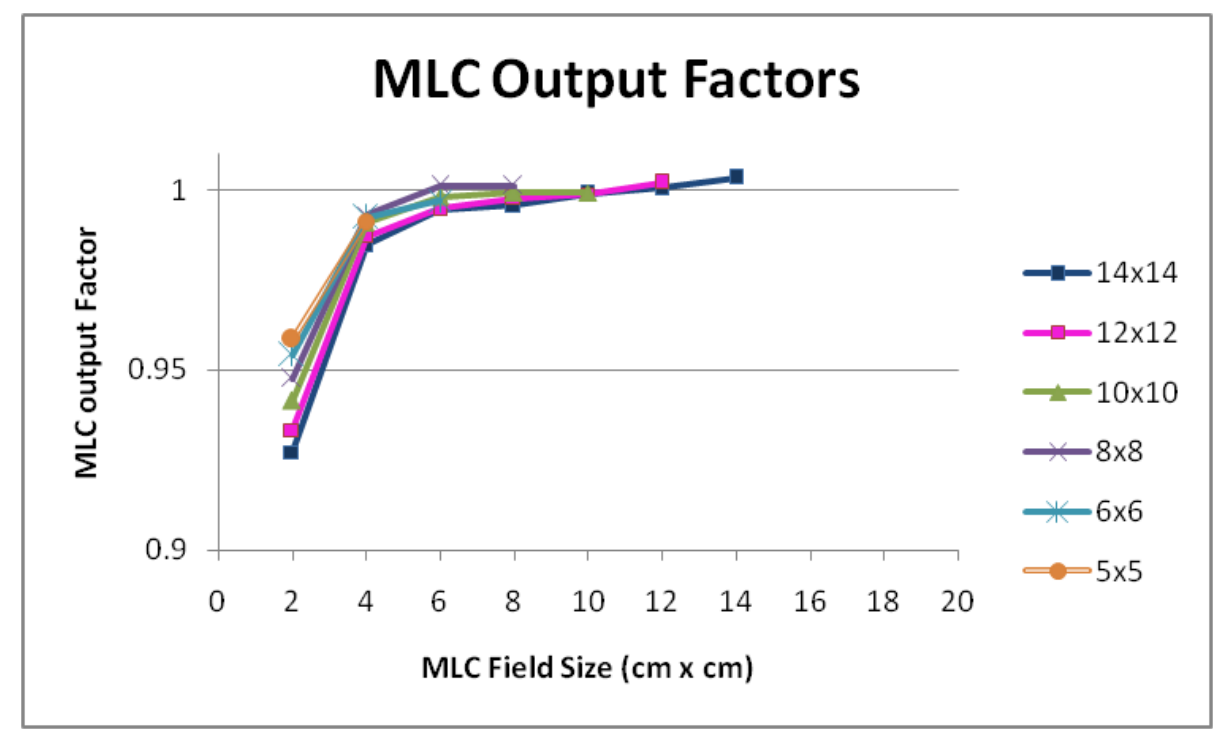

Figure 6: Output factors for Multi-Leaf Collimator (MLC) field sizes ranging from $2 \times 2 \mathrm{~cm}^{2}$ to 14 $\times 14 \mathrm{~cm}^{2}$. 
factors for various MLC field sizes.

Central Axis Profiles and Penumbra

The same dosimetric parameters used for conventional collimators were evaluated for MLC-shaped fields as well. Dose profiles obtained in crossplane (L-R) and inplane (G-F) directions across the central axis were analyzed for FWHM and penumbra measurements. The full width at half maximum (FWHM) was evaluated at $50 \%$ isodose line, defining the radiation field width and the $80 \%-20 \%$ width was considered for penumbra. The normalized profiles and the evaluated data through Monte Carlo simulations, IC profiler and Gafchromic films are presented in Figure 7 and Tables 2, 3 and 4.

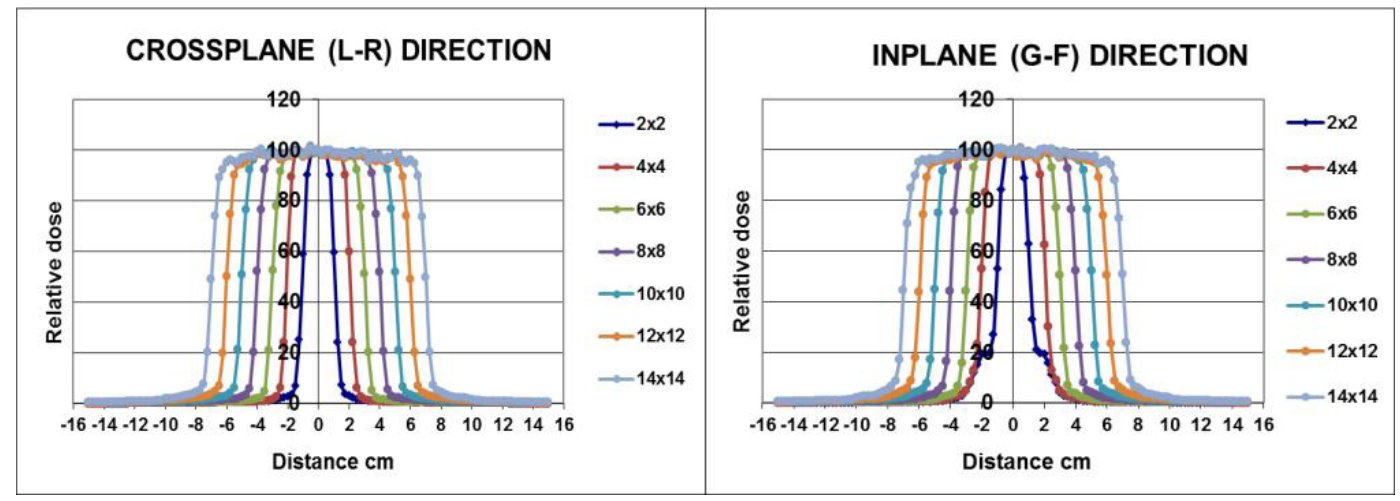

(A)

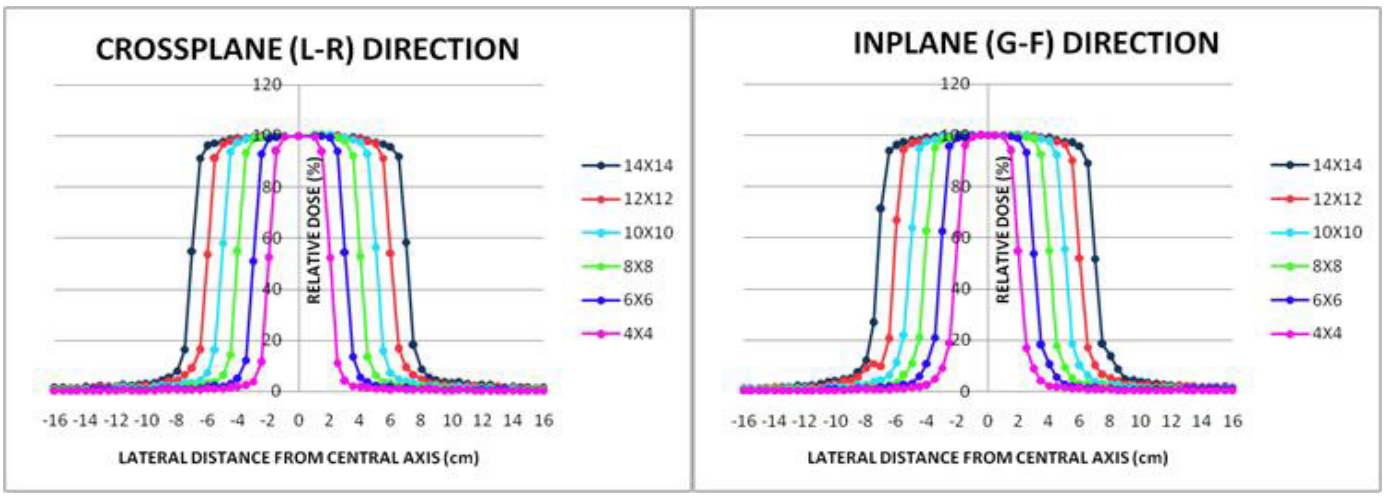

(B)

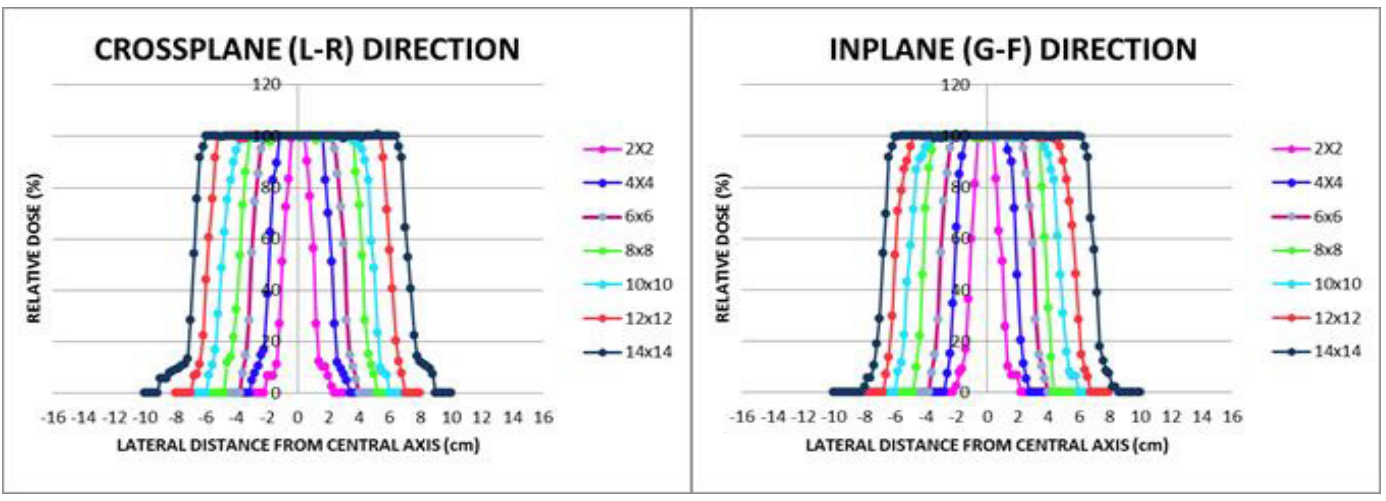

(C)

Figure 7: Crossplane and Inplane beam profiles for Multi-Leaf Collimator (MLC) generated square fields $-2 \times 2 \mathrm{~cm}^{2}$ to $14 \times 14 \mathrm{~cm}^{2}$ from (A) Monte Carlo simulations (B) Ionization chamber (IC) Profiler (C) Gafchromic films. 
Table 2: Analysis of dosimetric parameters- Full width at half maximum (FWHM) and Penumbra from Monte Carlo simulations.

FIELD PROFILE/ FWHM PENUMBRA PENUMBRA PENUMBRA PENUMBRA $(\mathrm{cm} \times \mathrm{cm})$ DIRECTION $(\mathrm{mm})$ Gantry $(\mathrm{mm})$ Feet $(\mathrm{mm}) \quad$ Left $(\mathrm{mm}) \quad$ Right $(\mathrm{mm})$

\begin{tabular}{|c|c|c|c|c|c|c|}
\hline \multirow{2}{*}{$2 \times 2$} & G-F & 21.3 & 7.0 & 8.3 & & \\
\hline & $L-R$ & 21.2 & & & 4.8 & 4.8 \\
\hline \multirow{2}{*}{$4 \times 4$} & G-F & 41.2 & 5.4 & 5.5 & & \\
\hline & $L-R$ & 41.3 & & & 4.8 & 4.7 \\
\hline \multirow{2}{*}{$6 \times 6$} & G-F & 59.6 & 5.2 & 5.7 & & \\
\hline & $L-R$ & 60.3 & & & 5.6 & 5.4 \\
\hline \multirow{2}{*}{$8 \times 8$} & G-F & 79.7 & 5.3 & 5.9 & & \\
\hline & $L-R$ & 80.3 & & & 5.6 & 5.9 \\
\hline \multirow{2}{*}{$10 \times 10$} & G-F & 99.7 & 5.5 & 6.0 & & \\
\hline & $L-R$ & 100.1 & & & 5.8 & 5.7 \\
\hline \multirow{2}{*}{$12 \times 12$} & G-F & 119.5 & 5.8 & 6.6 & & \\
\hline & $L-R$ & 119.9 & & & 5.8 & 6.0 \\
\hline \multirow{2}{*}{$14 \times 14$} & G-F & 139.5 & 6.3 & 6.8 & & \\
\hline & L-R & 139.9 & & & 6.0 & 6.0 \\
\hline
\end{tabular}

FWHM: Full width at half maximum

Table 3: Analysis of dosimetric parameters- Full width at half maximum (FWHM) and Penumbra using ionization chamber (IC) Profiler.

FIELD PROFILE/ FWHM PENUMBRA PENUMBRA PENUMBRA PENUMBRA $(\mathrm{cm} \times \mathrm{cm})$ DIRECTION $(\mathrm{mm})$ Gantry $(\mathrm{mm})$ Feet $(\mathrm{mm}) \quad$ Left $(\mathrm{mm}) \quad$ Right $(\mathrm{mm})$

\begin{tabular}{|c|c|c|c|c|c|c|}
\hline \multirow{2}{*}{$2 \times 2$} & G-F & 21.6 & 7.4 & 7.8 & & \\
\hline & $L-R$ & 21.3 & & & 9.6 & 9.2 \\
\hline \multirow{2}{*}{$4 \times 4$} & G-F & 41.6 & 7.5 & 7.7 & & \\
\hline & $L-R$ & 40.2 & & & 7.2 & 7.2 \\
\hline \multirow{2}{*}{$6 \times 6$} & G-F & 61.5 & 8.1 & 8.0 & & \\
\hline & $L-R$ & 60.1 & & & 7.4 & 7.4 \\
\hline \multirow{2}{*}{$8 \times 8$} & G-F & 81.4 & 8.1 & 8.0 & & \\
\hline & $L-R$ & 80.2 & & & 7.5 & 7.6 \\
\hline \multirow{2}{*}{$10 \times 10$} & G-F & 101.5 & 8.5 & 8.1 & & \\
\hline & $L-R$ & 100.8 & & & 7.6 & 7.6 \\
\hline \multirow{2}{*}{$12 \times 12$} & G-F & 121 & 7.6 & 8.2 & & \\
\hline & $L-R$ & 119.9 & & & 8.0 & 8.0 \\
\hline \multirow{2}{*}{$14 \times 14$} & G-F & 141.4 & 9.2 & 8.5 & & \\
\hline & L-R & 140.4 & & & 7.9 & 7.9 \\
\hline
\end{tabular}

FWHM: Full width at half maximum 
Table 4: Analysis of dosimetric parameters- Full width at half maximum (FWHM) and Penumbra using Gafchromic films.

FIELD PROFILE/ FWHM PENUMBRA PENUMBRA PENUMBRA PENUMBRA $(\mathrm{cm} \times \mathrm{cm})$ DIRECTION $(\mathrm{mm})$ Gantry $(\mathrm{mm})$ Feet $(\mathrm{mm}) \quad$ Left $(\mathrm{mm}) \quad$ Right $(\mathrm{mm})$

\begin{tabular}{|c|c|c|c|c|c|c|}
\hline \multirow{2}{*}{$2 \times 2$} & G-F & 20.5 & 6.2 & 5.4 & & \\
\hline & $L-R$ & 20.9 & & & 5.6 & 6.4 \\
\hline \multirow{2}{*}{$4 \times 4$} & G-F & 41.2 & 5.4 & 6.4 & & \\
\hline & $L-R$ & 40.6 & & & 5.0 & 5.0 \\
\hline \multirow{2}{*}{$6 \times 6$} & G-F & 60.8 & 6.2 & 6.4 & & \\
\hline & $L-R$ & 61.2 & & & 6.0 & 6.6 \\
\hline \multirow{2}{*}{$8 \times 8$} & G-F & 80.6 & 7.5 & 6.3 & & \\
\hline & $L-R$ & 80.5 & & & 5.3 & 5.4 \\
\hline \multirow{2}{*}{$10 \times 10$} & G-F & 99.6 & 8.8 & 6.2 & & \\
\hline & $L-R$ & 98.5 & & & 7.6 & 7.2 \\
\hline \multirow{2}{*}{$12 \times 12$} & G-F & 120 & 7.0 & 6.9 & & \\
\hline & $L-R$ & 117.9 & & & 7.5 & 8.5 \\
\hline \multirow{2}{*}{$14 \times 14$} & G-F & 140.8 & 5.6 & 7.8 & & \\
\hline & $L-R$ & 139 & & & 6.7 & 6.8 \\
\hline
\end{tabular}

FWHM: Full width at half maximum

Comparison of beam profile for a $10 \times 10$ $\mathrm{cm}^{2}$ MLC field at depth of maximum dose $\left(D_{\max }\right)$ for three modes of measurements is shown in Figure 8.

The Gafchromic film profiles show sharp dose fall-off than the MC simulations and IC profiler measurements. Despite this difference, there is good agreement between the simulated and measured data for $10 \times 10 \mathrm{~cm}^{2}$ MLC field size.

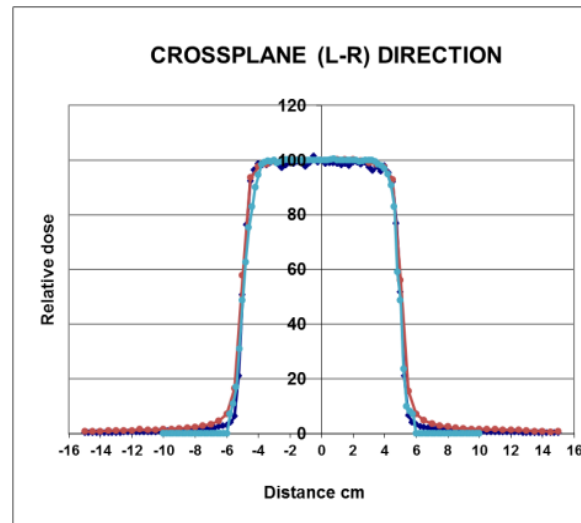

(A)

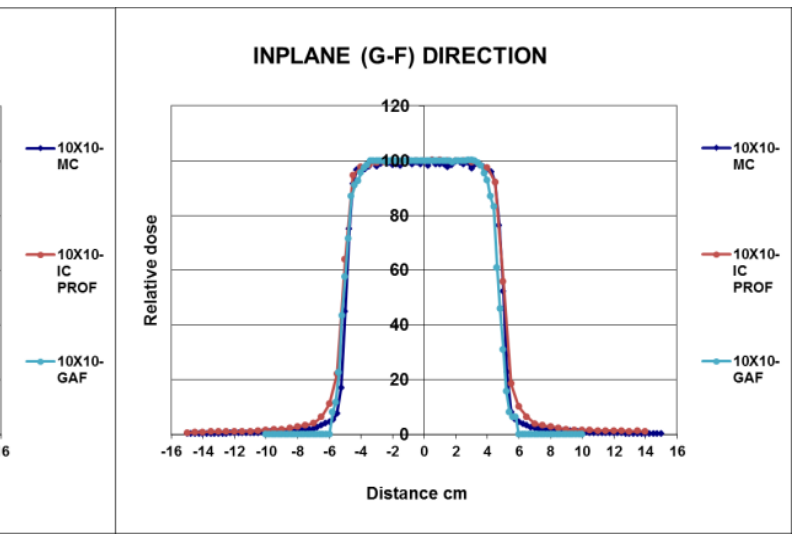

(B)

Figure 8: Comparison of $10 \times 10 \mathrm{~cm}^{2}$ Multi-Leaf Collimator (MLC) field beam profile (A) crossplane (B) inplane for Monte Carlo (MC) simulations, ionization chamber (IC) profiler and Gafchromic film measurements. 


\section{Discussion}

\section{Effect of Leaf Design}

The projected leaf width at isocenter due to $\mathrm{X}$-ray attenuation is dependent on the distance between source and MLC. In these prototypes, the source to diaphragm distance is $52 \mathrm{~cm}$ and the bottom of the leaves is at $56 \mathrm{~cm}$. The results presented in Figure 3 clearly show that the non-divergent prototype-1 MLC leaf casts a wider shadow $\approx 1.2 \mathrm{~cm}$ and hence has more width in the valley region of the curves. In view of the large leaf width and cobalt penumbra, the difference between the divergent and non-divergent leaf is small. As seen in Figure 4 , there is good agreement between the results obtained from Gafchromic film scans and the MC simulations.

\section{Treatment Planning Evaluation}

The $50 \%$ isodose line represents the region where $50 \%$ of the prescribed dose lies. This ensures that the periphery of the tumor will receive at least the prescribed dose. Moreover, the dose inside the tumor will be higher than the prescribed dose following rapid dose fall off outside the tumor thus sparing the organs at risk (OARs). Dosimetrically, the rounded ends of the leaves resulted in smooth isodose lines around the target.

\section{Radiation Characteristics of Pro- totype-2 Mlc \\ Leakage through Mlcs}

The leaf transmission plays an important role in determining the height of the MLC leaves. Measurements agree within recommended tolerance limit of $2 \%$ as per Task Group (TG)-50 reports. The leaf end transmission was found to be within 5\% tolerance limit, applicable for non-IMRT MLCs.

\section{Output Factors}

Since MLCs are used as tertiary collimator with existing collimator jaws, the output factors are not affected unless the MLC generated fields are smaller than the jaw opening. The scatter fluence depends mostly on the collimator opening. For every field, we first apply the collimator field size factor. In addition, we apply the MLC factor based on the equivalent square of the MLC open area.

\section{Central Axis Profiles and Penumbra}

The simulated as well as measured profile data in crossplane and inplane directions showed comparable results. Experimentally, an ion chamber array detector IC profiler was chosen since the available 3D water phantom was not suitable for dose measurements with MLCs in place on the telecobalt machine due to height limitations. Both IC profiler and Gafchromic films produced reliable data. From the tabulated data, it can be seen that FWHM for all the fields agree within $2 \mathrm{~mm}$ and penumbra lies within 5-10 $\mathrm{mm}$ range as mentioned in Atomic Energy Regulatory Board (AERB) safety code [17]. In case of measured data, it is observed that penumbra is little more significant in gantry to feet direction (G-F) since the jaws were opened wider than the MLC field sizes, which can be optimized with same jaw size in direction perpendicular to leaf movement.

\section{Conclusion}

In this study, the prototype MLC designed is a retrofit to existing cobalt machines. For convenience of easy fabrication, the leaves in the prototype-1 MLC were fabricated without divergence. Since straight blocks cause undesirable transmission penumbra in the target, the prototype- 2 MLC has been designed to follow divergence of the cobalt radiation with a step design for leaf sides and leaf end rounded edge. The use of divergent leaf is most useful in some clinical situations where there is open field on both sides of the leaf. Experimentally, the dosimetric parameters of divergent leaf design were found to be within tolerance limits. With these MLCs in place, the beam characteristics remain unaltered. Also, we can conclude that in view of providing cost-effective care with the existing telecobalt machines in 
rural areas, the prototype- 2 MLC system can be used for conformal therapy, eliminating the need for blocks and setting up the fields quickly.

\section{Authors' Contribution}

Akula Roopa Rani conceived the idea and introduction of the paper. As well, he has written the related literature and implemented the method. Results and Analysis was carried out by Akula Roopa Rani and Komanduri Ayyangar. A.R. Reddy, P. Yadagiri Reddy and A. Anil kumar helped writing the related works. The research work was proofread and supervised by Komanduri Ayyangar, A.R. Reddy and P. Yadagiri Reddy.

All authors read, modified, and approved the paper.

\section{Funding}

This study was supported by the Board of Research in Nuclear Sciences with grant number: 2010/36/65/BRNS.

\section{Conflict of Interest}

None

\section{References}

1. Munshi A, Ganesh T, Mohanti BK. Radiotherapy in India: History, current scenario and proposed solutions. Indian J Cancer. 2019;56(4):359-63. doi: 10.4103/ijc. IJC_82_19. PubMed PMID: 31607709.

2. Jayarajan K, Kar DC, Sahu R, Radke MG, Singh M. BARC develops cobalt-60 teletherapy machine for cancer treatment. BARC Newsletter; Mumbai: Bhabha Atomic Research Centre; 2005.

3. Ravichandran R. Has the time come for doing away with Cobalt-60 teletherapy for cancer treatments. J Med Phys. 2009;34(2):63-5. doi: 10.4103/09716203.51931. PubMed PMID: 20098538. PubMed PMCID: PMC2805891.

4. Van Dyk J, Battista JJ. Cobalt-60: An old modality, a renewed challenge. Curr Oncol. 1996;3:8-17.

5. Rani AR, Ayyangar K, Reddy AR, Kumar AA, Reddy PR. Design and development of an add-on automated multi leaf collimator for telecobalt therapy machine and study of its characteristics. Int $J$ Cancer Ther Oncol. 2018;6(1):19-30.

6. Galvin JM, Han K, Cohen R. A comparison of multileaf-collimator and alloy-block field shaping. Int $J$ Radiat Oncol Biol Phys. 1998;40(3):721-31. doi: 10.1016/s0360-3016(97)00815-8. PubMed PMID: 9486625 .
7. Galvin JM, Leavitt DD, Smith AA. Field edge smoothing for multileaf collimators. Int J Radiat Oncol Biol Phys. 1996;35(1):89-94. doi: 10.1016/s03603016(96)85015-2. PubMed PMID: 8641931.

8. Ayyangar KM, Reddy AR. Development of an automated MLC for telecobalt therapy machine with a potential for conformal therapy. Proceedings of 32nd Annual conference of Association of Medical Physicists of India; India: CMCH Vellore; 2011.

9. Akula RR, Anil Kumar A, Talluri AK, Sresty M, Rao CR, Sankaranarayana $\mathrm{M}$, et al. Calibration measurements on the prototype MLC for cobalt 60 teletherapy machine. Proceedings of 33rd Annual conference of Association of Medical Physicists of India; India, Mangalore: Association of Medical Physicists; 2012.

10. Roopa Rani A, Ayyangar KM, Reddy AR, Anil Kumar A, Yadagiri Reddy PR. Experimental verification of Monte Carlo simulations of MLC fields for Cobalt- 60 therapy machine. Proceedings of 35th Annual Conference of Association of Medical Physicists of India; India, Loni: Association of Medical Physicists; 2014. p. 105.

11. Ayyangar KM, Rani RA, Kumar A, Reddy AR. Monte Carlo study of MLC fields for cobalt therapy machine. J Med Phys. 2014;39(2):71-84. doi: 10.4103/09716203.131279. PubMed PMID: 24872604. PubMed PMCID: PMC4035619.

12. Boyer A, Biggs P, Galvin J, Klein E, Losasso T, Low $D$, et al. Basic applications of multileaf collimators. AAPM Report NO. 72; United States: American Association of Physicists in Medicine; 2001.

13. Kumar AA, Akula RR, Ayyangar K, Krishna RP, Vuppu S, Narayana PV, Rao AD. In-house quality check of external beam plans using 3D treatment planning systems - a DVH comparison. J Appl Clin Med Phys. 2016;17(3):138-46. doi: 10.1120/jacmp. v17i3.6020. PubMed PMID: 27167271. PubMed PMCID: PMC5690922.

14. Krishna GS, Akula RR, Kumar AA, Srinivas V, Ayyangar K, Reddy PY. DVH Analysis of Cobalt-60 treatment plans incorporating a recently developed MLC. Int J Cancer Ther Oncol. 2016;4(3):431. doi: 10.14319/IJCTO.43.1.

15. Niroomand-Rad A, Blackwell CR, Coursey BM, et al. Radiochromic film dosimetry: recommendations of AAPM Radiation Therapy Committee Task Group 55. American Association of Physicists in Medicine. Med Phys. 1998;25(11):2093-115. doi: 10.1118/1.598407. PubMed PMID: 9829234.

16. International Electro technical Commission. Part2: Particular requirements for the safety of gamma beam therapy equipment. Report No. IEC-606012-11; Geneva: IEC; 1997.

17. Atomic Energy Regulatory Board. AERB safety code for radiation therapy sources, equipment and installations. AERB SAFETY CODE NO. AERB/RF-MED/SC-1 (Rev. 1); India: AERB; 2011. 